\title{
La enseñanza Secundaria Básica y su vinculación con el principio de estudio - trabajo en la pedagogía cubana
}

\author{
Basic Secondary education and its link with the principle of \\ study - work in Cuban pedagogy
}

\author{
Bárbara Companioni Turiño ${ }^{1}$ \\ bcompanioni@ucf.edu.cu \\ www.orcid.0000-0001-7029-4911
}

Recibido: 1/4/2020, Aceptado: 1/6/2020

\begin{abstract}
RESUMEN
El objetivo de este trabajo es presentar una reflexión acerca del trabajo y la educación secundaria, tema que encuentra en los países de la región un marcado interés, sobre todo por la importancia que se le asigna a este nivel educativo en el marco de los procesos de modernización y desarrollo. El modelo de educación secundaria debe dar respuesta a quienes vivirán y actuarán en una realidad futura distinta. Se hace una valoración acerca del papel del estudio y el trabajo, sobre todo, de su integración en el proceso formativo y cómo se convirtió para la educación cubana en un principio rector, donde la inserción de la juventud a la actividad laboral productiva ha tenido un significado y sentido trascendental en el modelo educativo. Se ha considerado todo lo que encierra en el orden formativo y en el desarrollo de la personalidad de cada individuo. La vinculación estrecha entre el estudio - trabajo define entonces dos objetivos esenciales. El formativo, que tiene como propósito esencial desarrollar la conciencia del productor de bienes sociales y el segundo, lograr que, mediante la práctica laboral, los alumnos se pongan en contacto directo con la realidad que los rodea. Todo esto es decisivo en la formación de una concepción científica del mundo, como también lo es la posibilidad de establecer la relación de los alumnos con la clase obrera y conocer sus tradiciones laborales, lo cual contribuye al desarrollo del sistema de actitudes y valores ciudadanos. Se concluye que, habiendo tenido una buena experiencia en décadas pasadas, hay que retomar y fortalecer esta metodología de enseñanza.
\end{abstract}

Palabras clave: enseñanza Secundaria Básica, vinculación con el principio de estudio - trabajo

\section{ABSTRACT}

It is reflected in the work on secondary education that finds in each of the countries of the region a marked interest, above all because of the importance assigned to this educational level in the framework of modernization and development processes; she must give an answer in the present to who will live and act in a different future reality. An assessment is made about the role of study and work, above all, its integration

\footnotetext{
${ }^{1}$ Universidad de Cienfuegos, Cuba.
} 


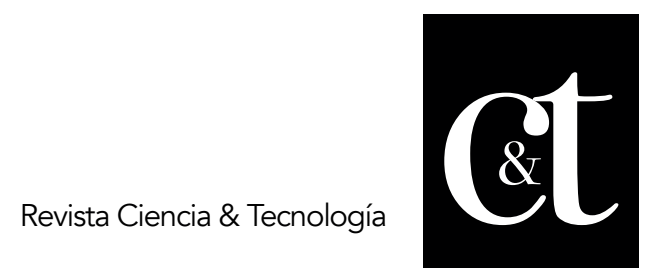

No. 27, 31 de julio de 2020

ISSN impreso: 1390 - 6321

ISSN online: 2661 - 6734

into the training process and how it became a guiding principle for Cuban education. Therefore, the insertion of youth into productive work activity has had a transcendental meaning and sense in the educational model, everything that is included in the training order and in the development of the personality of each individual has been considered. The close link between study-work then defines two essential objectives: the training, that has the essential purpose of developing the consciousness of the producer of social goods and, second, ensuring that, through work practice, students get in direct contact with the reality that surrounds; all of which is decisive in the formation of a scientific conception of the world, as is the possibility of establishing the relationship of students with the working class and knowing their labor traditions, which contributes to the development of the system of attitudes and values citizen. Tis work concludes emphasizing the importance of this issue, which has to be retaken and strengthened.

Keywords: Basic Secondary education, connection with the principle of study - work

\section{Introducción}

Los análisis internacionales relacionados con la secundaria básica, tienden a acusar a este tramo educativo de ser inequitativo, en el cual se prepara mal a los alumnos, tanto para los estudios superiores como para la entrada de éstos al mercado laboral, al mantenerse separado del mundo exterior. Hoy se reconoce la imposibilidad de proporcionar a los alumnos "todos" los conocimientos y habilidades que le serán necesarios dentro de unos años; no solo por el crecimiento de las demandas sociales, sino también por el influjo del desarrollo de la ciencia y la tecnología. Todo ello está condicionando el cambio de los sistemas educativos, y su orientación para responder, de manera apropiada, ante la necesidad de proporcionar estrategias de aprendizaje, de forma que puedan utilizarlas de manera autónoma y flexible.

Es evidente que el diseño de las estrategias de aprendizaje no se desarrolla al margen de los contenidos, es bien importante la selección de los ejes cognitivos que sostienen las distintas actividades formativas en la escuela, su jerarquía y trascendencia. En este punto, las asignaturas no son enseñadas por derecho propio, sino por la contribución específica de cada una a la formación cultural, social e integral de los alumnos, lo cual responde al encargo social general de la educación (Ruiz, 2008).

Desde el I Perfeccionamiento Educacional, iniciado en la década del 70 y más concretamente durante el II Perfeccionamiento en la década del 80, la escuela secundaria se ha orientado hacia la formación básica e integral del alumno cubano, sobre la base de una cultura general, con una sólida formación científica para explicar y comprender los problemas de la vida diaria. Hoy la orientación formativa de la escuela pone el énfasis en la posibilidad de que el adolescente llegue "a estar plenamente identificado con su nacionalidad y patriotismo, al conocer y entender su pasado, enfrentar su presente y su preparación futura, adoptando conscientemente la opción del socialismo, que garantice la defensa de las conquistas sociales y la continuidad de la obra de la Revolución, expresado en sus formas de sentir, de pensar y de actuar $^{2} "$.

\footnotetext{
${ }^{2}$ Lizardo García "Propuesta curricular para la Secundaria Básica actual. Fundamentos teóricos metodológicos", Pág. 41. 2003.
} 


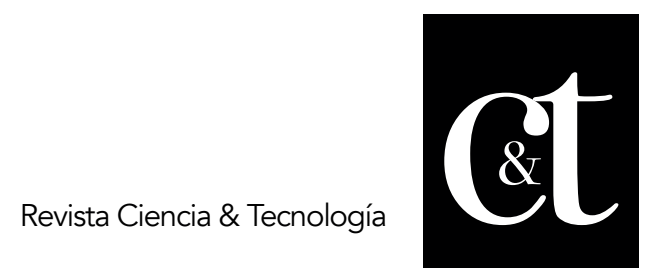

No. 27, 31 de julio de 2020

ISSN impreso: 1390 - 6321

ISSN online: 2661 - 6734

Dentro de sus objetivos formativos generales, no se ha renunciado a la formación para la vida $^{3}$ lo cual explica que de manera coincidente se refiera a contenidos esenciales relacionados con la necesidad de lograr que los alumnos demuestren una correcta actitud hacia el medio ambiente, expresado en su modo de actuación en relación con la protección el ahorro de recursos, fundamentalmente energéticos y el cuidado de la propiedad social, desarrollando, además "... una cultura laboral y tecnológica alcanzada a través del desarrollo de habilidades y capacidades generales, politécnicas y laborales que le permitan, desde la vinculación activa y consciente del estudio con el trabajo emplearlas de manera útil en la solución de problemas de la vida cotidiana, con la utilización de objetos como; los mecanismos, las máquinas, los sistemas y los medios para operar con los materiales, la energía y la información, con una conciencia de productores y orientada por el sistema de valores desarrollado tanto en las clases como en la experiencia cotidiana, poniendo de manifiesto la lógica del pensamiento y modos de actuación propios de la actividad laboral ${ }^{4}$.

Desde este referente, se entiende que la vida de la escuela secundaria básica, aún en la concepción renovadora de la transformación actual, debe caracterizarse por un clima favorable hacia el estudio y el trabajo: esto significa que docentes y alumnos participen conscientemente en la formación de valores y actitudes laborales desde el estudio mismo de las asignaturas o desde las vivencias que proporcionan las actividades extradocentes o extracurriculares, al ejecutar de manera protagónica, su diseño, concreción y control. En este sentido, se advierte que, como consecuencia de esta necesidad, resulta imprescindible establecer un estilo pedagógico científico a partir de formas de trabajo grupal e individual, lo cual garantice el desarrollo de las capacidades intelectuales, manuales y contribuya a la formación de orientaciones valorativas éticas, morales, sobre la base de las necesidades individuales, sociales y del desarrollo alcanzado por ellos.

Para lograr el fin, los objetivos del nivel y de cada uno de los grados, la escuela secundaria básica organiza y ejecuta un sistema de actividades, las cuales se pueden agrupar en dimensiones tales como, políticas, ideológicas, docentes, laborales, deportivas, culturales, recreativas, vocacionales y patrióticos - militares. Con independencia de estas clasificaciones de las actividades en tales dimensiones, resulta necesario partir del criterio general de que en la escuela todas las actividades deben instruir y educar, es por eso que cada aula deberá convertirse en un verdadero taller de construcción del conocimiento, de creación, de laboriosidad y respeto, partiendo de las experiencias y vivencias de cada uno de los alumnos.

\section{Desarrollo}

La esencia de las transformaciones educativas está en situar al hombre como objeto real del cambio, como protagonista principal, y a las estructuras de dirección con nuevos métodos y estilos de trabajo que se deben emplear como medio para lograr tales fines. Por tanto, una de las exigencias a cumplimentar en esas transformaciones se relaciona con el establecimiento de condiciones sociales y pedagógicas, cada vez

\footnotetext{
${ }^{3}$ Véase objetivos generales, Versión 7 del "Modelo de escuela" para la secundaria básica, Proyecto, 29 de abril 2003.

${ }^{4}$ Lizardo García: "Propuesta curricular para la escuela Secundaria Básica actual. Fundamentos teóricos y metodológico", p 42
} 


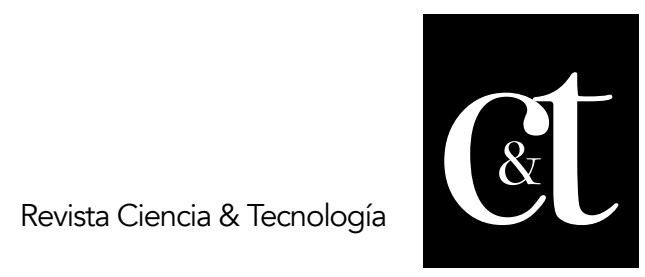

No. 27, 31 de julio de 2020

ISSN impreso: 1390 - 6321

ISSN online: 2661 - 6734

más cercanas al profesor y al alumno, con una labor más creadora y ajustada a las condiciones concretas de cada localidad.

Para lograr este propósito, se hace necesario elevar la responsabilidad de los docentes, promover su reflexión, determinar criterios y errores, revelar la necesidad que tienen de realizar modificaciones y, en consecuencia, lograr su implicación para accionar en el cambio de sus puntos de vista, estilos de trabajo y modos de actuación. Por lo anteriormente expuesto, ubicamos al profesor, como es lógico, en la escuela por ser ésta el núcleo básico hacia el cual se dirigen las transformaciones, y de ellos se erigieron, por excelencia, los centros de referencia, que se convirtieron en el vehículo más eficaz para entrenar a dirigentes, a funcionarios, docentes, tanto en los conceptos que sustentaron los cambios, así como, en su aplicación práctica.

Una escuela con estas características exige aprovechar en el proceso de enseñanza las condiciones del territorio donde se encuentra enclavada. En este proceso debe estar dotada de cierta estabilidad, condiciones y posibilidades para conducir el trabajo metodológico que constituye el soporte material para demostrar los niveles cualitativos que pueden y deben lograrse. Dentro de sus funciones, se destaca la de ejercer una acción educativa que llegue a todos los ciudadanos de la comunidad, con el fin de involucrarlos en el proceso y así aprovechar las mejores experiencias en el proceso pedagógico.

Es importante destacar que, para lograr un cambio efectivo en la práctica educativa de la escuela, es preciso dar un tratamiento particular al colectivo pedagógico, promover su cohesión e intereses profesionales y generales. Se debe seguir como eslabón importante la búsqueda de la implicación de los docentes en el proceso de cambio, donde debe existir una comunicación pedagógica apropiada que estimule el autoconocimiento. En la escuela a la que se aspira es imprescindible lograr una acertada dirección del cambio, y partir del criterio de que esta institución opera como centro transformador y desarrollador de todos los agentes educativos, los cuales participan en la socialización de los alumnos.

En este tipo de centro se advierte la importancia que tiene la formación en y para el trabajo. Se superan las concepciones de la orientación vocacional para acentuar el interés en las actividades y los conocimientos vinculados con el trabajo en su amplia acepción, lo cual debe permitirle al alumno poder alimentarse, vestirse, garantizar y satisfacer las necesidades perentorias de la vida humana. De forma general, la escuela debe preparar jóvenes amantes del trabajo, portadores de sólidos sentimientos de respeto hacia la creación y el resultado del esfuerzo de los hombres, así como, reconocer la fuerza defensiva y desarrollo de la labor del hombre cuando crea y aprovecha los recursos que tienen a su disposición para vivir y ayudar al colectivo al que pertenece.

La experiencia cubana, en torno a la educación del alumno desde la escuela secundaria, ha privilegiado la valoración del trabajo como actividad formativa por excelencia y esto explica que un conjunto de personas, nacidas en fechas próximas, las cuales hayan recibido educación e influjos culturales y sociales semejantes se comporten de manera afín o comparable en algunos sentidos; de ahí que tengan en su historia de vida, fuertes vivencias relacionadas con la actividad laboral sobre todo en la agrícola. 


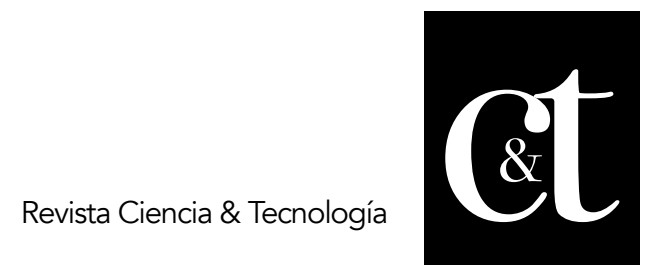

No. 27, 31 de julio de 2020

ISSN impreso: 1390 - 6321

ISSN online: 2661 - 6734

Las potencialidades de este tipo de actividad laboral se han concretado con la acreditación de etapas curriculares dedicadas a ella, con lo cual se debe cumplimentar el vínculo entre el estudio y el trabajo. Este principio de la pedagogía cubana constituye el principal interés que ha motivado esta investigación, siendo premisa base para comprender las concepciones que necesitan asumirse en el marco de las transformaciones, las cuales han comenzado a tener lugar en este nivel educativo y están definiendo el perfil de formación del alumno y el modelo de escuela secundaria

\section{El vínculo estudio -trabajo. Principio de la Pedagogía cubana}

La idea de educar en el trabajo aparece expresada claramente por los fundadores del socialismo científico. Carlos Marx fundamentó la necesidad de combinar el estudio con el trabajo, por lo que la práctica significa en la transformación de la sociedad, y definió la educación del futuro como aquella. "en la que se combinará para todos los chicos a partir de cierta edad el trabajo productivo con la enseñanza y la gimnasia no solo como método para intensificar la producción social, sino también como el único método que permite producir hombres plenamente desarrollados ${ }^{5}$."

También Lenin, en su lucha por la formación de la juventud, señaló las vías para la incorporación al trabajo socialmente útil como elemento indispensable en su educación. Le concedió gran importancia a la participación activa de la juventud en la reconstrucción económica del país.

El héroe nacional cubano, José Martí, con sus convicciones avanzadas, defendió siempre la idea de que "el hombre crece con el trabajo que sale de sus manos "y el líder revolucionario Fidel Castro, -exponente esencial de la simbiosis del pensamiento marxista-leninista y martiano- ha expresado que "el trabajo ha de ser el pedagogo de la juventud". De hecho, la valoración acerca del papel del estudio y el trabajo, sobre todo, de su integración en el proceso formativo se convirtió para la educación cubana en un principio rector. Por tanto, la inserción de la juventud (alumnos) a la actividad laboral productiva ha tenido un significado y sentido trascendental en el modelo educativo, se ha considerado todo lo que encierra en el orden formativo y en el desarrollo de la personalidad de cada individuo.

Convertir la combinación e integración del estudio con el trabajo como principio fundamental de nuestra educación encierra, en sí otros elementos o principios esenciales entre los que se distinguen: la relación de la escuela con la vida, la enseñanza de la teoría con la práctica y la fusión del trabajo intelectual con el manual. La vinculación estrecha entre el estudio - trabajo define dos objetivos esenciales, el formativo que tiene como propósito esencial desarrollar la conciencia del productor de bienes sociales y lograr que, mediante la práctica laboral, los alumnos se pongan en contacto directo con la realidad que los rodea; todo lo cual es decisivo, en la formación de una concepción científica del mundo, como también lo es la posibilidad de establecer la relación de los alumnos con la clase obrera y conocer sus tradiciones laborales, lo cual contribuye al desarrollo del sistema de actitudes y valores ciudadanos. A ello se agrega, el objetivo económico que persigue el propósito de integrar a la producción, y al trabajo social la fuerza laboral de los alumnos con una orientación productiva / educativa que permita una participación activa en la creación de bienes materiales. Pero estos objetivos no se dan aisladamente, sino que interactúan complementándose (Guibo y Sabó, 2007).

${ }^{5}$ Carlos Marx. "El Capital" t,1, p,434 


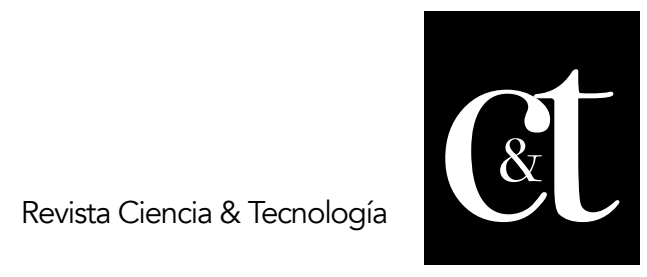

No. 27, 31 de julio de 2020

ISSN impreso: 1390 - 6321

ISSN online: 2661 - 6734

Para que el trabajo resulte verdaderamente formativo, éste debe tener un valor social y responder a una necesidad real, saber qué hacer, cómo hacerlo y cuál es el beneficio que su labor brinda, además de conocer algunos parámetros, como valorar los niveles de producción y su calidad como productor. Esto indiscutiblemente desarrolla cualidades como el sentido del deber, la responsabilidad, la valoración de los demás y la autovaloración.

El trabajo que desarrolla el alumno ha de contribuir a la formación de hábitos y habilidades laborales, a la comprensión de los fenómenos, regularidades y relaciones del mundo natural lo que permitirá actuar en la transformación y el aprovechamiento de los recursos naturales, mediante la correcta planificación de la técnica y la ciencia. Todo proceso laboral debe responder a exigencias organizativas, por tanto, se debe planificar con el fin de garantizar el aseguramiento material de la producción y de la productividad, también, es necesario tener en cuenta que la distribución y dosificación del trabajo de los alumnos debe crear las condiciones de vida, la seguridad e higiene laboral que correspondan con lo que han de realizar. Siempre que el trabajo sea una actividad colectiva la cual brinde la posibilidad de ayuda y cooperación, manteniendo la responsabilidad individual, se contribuirá al desarrollo del colectivismo y la responsabilidad social.

Existen diferentes formas y modalidades de combinar el estudio con el trabajo en los distintos niveles de enseñanza:

Particularmente, la combinación del estudio con el trabajo en el nivel medio se organiza en dos enseñanzas: las secundarias básicas e institutos Preuniversitarios. En la secundaria la asignatura Educación Laboral desarrolla habilidades prácticas, así como la formación de hábitos de trabajo. En esta enseñanza también se facilita la posibilidad de que todas las asignaturas y actividades escolares contribuyan a la educación laboral de los alumnos, y con ello se consiga un nivel de desarrollo integral de la personalidad. Históricamente, el trabajo productivo agrícola se aplica en dos modalidades la escuela al campo y en el campo, esta se ha organizado a través de los huertos escolares, las labores de jardinería y otras actividades asociadas al trabajo agrícola donde los alumnos pueden realizar tareas de cultivo, atención cultural a las plantas, recogida de producción y a través de ello adquirir nociones generales del proceso. Esta concepción no pretende alcanzar un nivel de especialización agrícola pero los alumnos tienen la posibilidad de conocer el cultivo y participar activamente en la producción social.

\section{Resultados}

La práctica educativa desarrollada en Cuba, también legitima el valor del trabajo socialmente útil. Este se puede desarrollar dentro o fuera de la escuela y poseer diferentes contenidos como son: censos de población, limpieza, ordenamiento y embellecimiento, autoservicio en comedores escolares- pero el objetivo es el mismo: fortalecer la conciencia social de los alumnos, desarrollar en ellos los hábitos y habilidades necesarios para valerse por sí mismos y lograr la cooperación y el bienestar de los demás.

De la experiencia pedagógica y social acumulada, es posible precisar que, desde el punto de vista organizativo, la actividad laboral en la que participan los alumnos en los diferentes niveles suele ser muy semejante; se destaca una connotación curricular que patentiza su papel en el logro de los objetivos de formación, en unos casos como actividad extraescolar y en otros como espacio formativo esencial dentro del currículo 


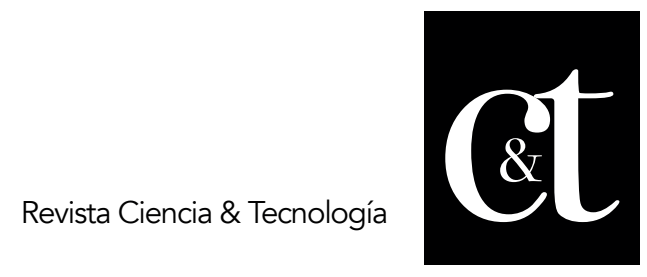

No. 27, 31 de julio de 2020

ISSN impreso: 1390 - 6321

ISSN online: 2661 - 6734

que acredita el nivel alcanzado. Es evidente que en la educación secundaria las actividades de este tipo están más relacionadas con las labores agrícolas en las que el trabajo tiene un énfasis ético-social más que productivo, aun cuando en ellas pueden descansar los resultados de la producción.

Hay que reconocer que las labores de recolección, limpia de campo, guataqueo, chapea, siembra, abono y escarde entre otras, a las que se han dedicado los alumnos de secundaria básica en las modalidades de escuela al campo y en el campo resultan vitales para la eficiencia productiva. Sin embargo, en lo que al aprendizaje se refiere no se ha logrado rebasar el conocimiento de las operaciones concretas que realizan; ya que las ven aisladas de un proceso productivo, solo tienen una visión parcial y estrecha lo cual deja de contribuir a la creación de intereses y a fortalecer la vinculación de la teoría y la práctica6.

Desde el punto de vista formativo, es el trabajo quien juega una influencia positiva en la formación de cualidades personales como por ejemplo la disciplina, la responsabilidad, la aceptación de la crítica y las relaciones en el colectivo de trabajadores durante la actividad laboral, y es mediante el trabajo que el alumno reconoce su valor e importancia formándose en ellos una conciencia de productores. El estudio realizado por la Dra. Josefina López Hurtado (1990), pone de manifiesto que los alumnos tienen más conciencia del valor social de su trabajo que un conocimiento del valor económico de la actividad que realizan.

Una perspectiva económica del Sistema de Estudio y Trabajo, aplicada en Cuba, advierte que su concreción ha estado sujeta a las necesidades socio productivas y a las demandas de fuerza de trabajo. La utilización y distribución de los alumnos como fuerza de trabajo ha descansado en las necesidades de la producción y la incidencia de esta actividad en la educación de los alumnos es mayor en aquellas escuelas que están ubicadas generalmente en el área productiva; así mismo, los análisis acerca de la relación entre la inversión y los gastos económicos en el empleo de este tipo de fuerza laboral no siempre han considerado el aporte formativo, en muchos casos, la producción de los alumnos arrojó saldos favorables en las primeras décadas (60 y 70).

En los últimos años, el riesgo de la eficiencia económica de las empresas involucradas deterioró la demanda de esta fuerza de trabajo, se argumentaron, por un lado, las limitaciones de recursos para asegurar, de manera mínima, las condiciones de vida y por otro el proceso de perfeccionamiento empresarial en que se han visto involucradas las empresas agropecuarias. Por la revisión de la bibliográfica realizada podemos plantear que en los años 79 y 80 lo producido por los alumnos fue superior a lo invertido, aunque el aseguramiento a la actividad laboral en estas fechas comenzó a presentar múltiples dificultades, lo cual limitó el valor formativo con que se concibieron las etapas de escuela al campo y las escuelas en el campo. ${ }^{7}$

\section{Conclusiones}

En el contexto histórico social del perfeccionamiento en la educación, se enfrentan hoy cambios sustanciales en el modelo educativo de la enseñanza secundaria básica,

\footnotetext{
6 Véase Estudio del comportamiento de la combinación del estudio con el trabajo en la Educación General. C. Doctora Josefina López Hurtado. (1990).

7 Véase Estudio y trabajo. Editorial Pueblo y Educación, 1990 Pág. 16 y 17.
} 


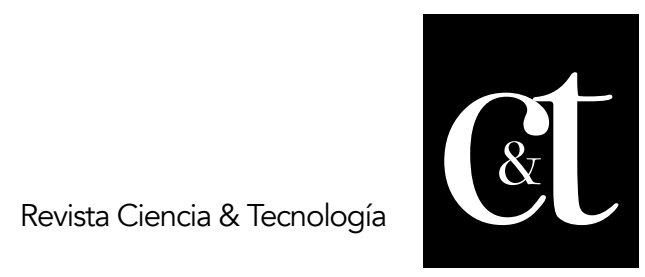

No. 27, 31 de julio de 2020

ISSN impreso: 1390 - 6321

ISSN online: 2661 - 6734

para el logro de una cultura general integral de los alumnos, como expresión de la Revolución Educacional en el país.

La experiencia educativa revelada, en lo concerniente al papel, de la combinación del estudio con el trabajo, alcanza su concreción en la actividad agrícola como una vía esencial para la preparación de hombres laboriosos, con mentalidad de productores, optimistas, creadores y sanos moral y físicamente, lo cual trasciende y rectora a la Pedagogía cubana. Se ha evidenciado que es un proceso que da buenos resultados, como se comprobó en las décadas de los setenta y ochenta, que si bien se vio afectado por una serie de problemas, sin embargo hay que replantear la metodología y aprender de esas experiencias.

\section{Referencias bibliográficas}

Concepción Marxista Leninista acerca de la enseñanza politécnica y la combinación del estudio con el trabajo. -La Habana: MINED, 1976.

Companioni Turiño, B. Ponencia para el examen de mínimo en problemas sociales de la ciencia. La agricultura urbana: una solución de un problema social desde la sustentabilidad. Universidad de Cienfuegos, 2002.

García, L. (2003). "Propuesta curricular para la Secundaria Básica actual. Fundamentos teóricos metodológicos", pp. 41, 42.

Guibo, A. y Sagó, M. (2007). La integración estudio-trabajo en el trabajo metodológico interdisciplinario del departamento Ciencias Naturales del Preuniversitario en el Campo EduSol, vol. 7, núm. 19, abril-junio, 2007, pp. 1-11 Centro Universitario de Guantánamo Guantánamo, Cuba

López Hurtado, J. (1990). Estudio del comportamiento de la combinación del estudio con el trabajo en la Educación General.

Marx, Carlos. (2000). El Capital. Tomo1. La Habana:Cuba.

Ruiz, J. (25/02/2008). El estudio-trabajo como principio pedagógico en la formación de profesionales. Educaweb. Recuperado de: https//:www.educaweb.com/noticia/2008/02/25/estudio-trabajo-comoprincipio-pedagogico-formacion-profesoniales-2813/ 